【研究简报】

\title{
$\mathrm{La}^{+}+\mathrm{NH}_{3} \rightarrow \mathrm{La}^{+} \mathrm{NH}+\mathrm{H}_{2}$ 反应的从头算研究
}

\author{
朱荣顺 戴树珊 * 叶 松 \\ (云南大学化学系, 昆明 650091 ． 联系人)
}

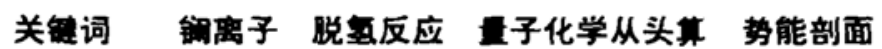

近些年, 过渡金属离子与一些小分子的气相反应研究是一相当活跃的领域 ${ }^{[1 \sim 3]}$. Clemmer 等实验研究了 $\mathrm{Sc}^{+}, \mathrm{Ti}^{+}, \mathrm{V}^{+}$与氨的反应 ${ }^{[4,5]}$. 对脱 $\mathrm{H}_{2}$ 反应: $\mathrm{M}^{+}+\mathrm{NH}_{3} \rightarrow \mathrm{M}^{+} \mathrm{NH}+\mathrm{H}_{2}$, 主 要是通过氧化加成一个 $\mathrm{N}-\mathrm{H}$ 键形成中间体 $\mathrm{H}-\mathrm{M}^{+} \mathrm{NH}_{2}$, 此中间体对 $\mathrm{Sc}^{+}$而言是单重态的, 对 $\mathrm{Ti}^{+}$而言是双重态．参与反应的离子,当它们的电子态不同,其反应活性也不同, 他们用“自旋 守恒”概念来解释. 自旋守恒的关键是考虑插入中间体 $\mathrm{H}-\mathrm{M}^{+}-\mathrm{NH}_{2}$, 比如: $\mathrm{V}^{+}$参加的反应, $\mathrm{V}^{+}$的 4 个价电子 $\cdots-\mathrm{NH}_{2}$ 的基态必定为三重态, 这样, $\mathrm{V}^{+}$的激发态 $\mathrm{a}^{3} \mathrm{~F}$ 比其基态 $\mathrm{a}^{5} \mathrm{D}$ 反应活 性高, 因为激发态 $\mathrm{a}^{3} \mathrm{~F}$ 与比较稳定的中间体保持了相同的自旋.

本文用从头算方法研究了 $\mathrm{La}^{+}+\mathrm{NH}_{3} \rightarrow \mathrm{La}^{+} \mathrm{NH}+\mathrm{H}_{2}$ 的单态和三重态势能剖面, 并对两种 势能剖面作了比较, 结果表明: 反应经过单重态中间体 $\mathrm{H}-\mathrm{La}^{+} \mathrm{NH}_{2}$, 接着通过四中心过渡态 TS2 的途径为能量最低途径,证实了 Clemmer 等“自旋守恒”的正确性, 并与 $\mathrm{Sc}^{+}-\mathrm{NH}_{3}$ 体系 ${ }^{[6]}$ 结果相类似.

\section{1 计算方法及过程}

（1）对反应物、产物、中间体及相应过渡态完成了解析梯度方法的全优化, 重原子 La 用 Christiansen 等人 ${ }^{[7]}$ 的平均相对论嗐势, 主要考虑其最外层的 $655 \mathrm{~d}$ 价电子, 相应的分裂价基为 $(3 \mathrm{~s} 3 \mathrm{p} 4 \mathrm{~d} / 2 \mathrm{~s} 2 \mathrm{p} 2 \mathrm{~d}), \mathrm{N}, \mathrm{H}$ 原子用 6-31G ** .

(2) 为了得到改进能量及考虑电子相关效应, 完成了二级 M $\Phi$ LLER-PLESSET 微扰单点 计算. 优化所得的过渡态都进行了振动分析, 在过渡态时其力常数矩阵有且仅有一个负本征 值, 证实他们为真实的过渡态. 所有计算均采用 Gamess 量化从头算程序 ${ }^{[8]}$.

\section{2 计算结果及讨论}

标题反应单态及三重态势能剖面见 图 1, 全优化所得稳定点及过渡态构型 列于图 2.

\section{1 单态势能面}

从图 1 可见, $\mathrm{La}^{+}$与 $\mathrm{NH}_{3}$ 通过长程 离子-诱导偶极力形成碰撞络合物 $\mathrm{La}^{\prime}$ $\mathrm{NH}_{3}$ (Complex), 这是反应的第一步, 此 过程没有能垒, 然后碰撞络合物 $\mathrm{La}^{+}$. $\mathrm{NH}_{3}$ 的一个 $\mathrm{H}$ 原子经过过渡态 TS 1 从

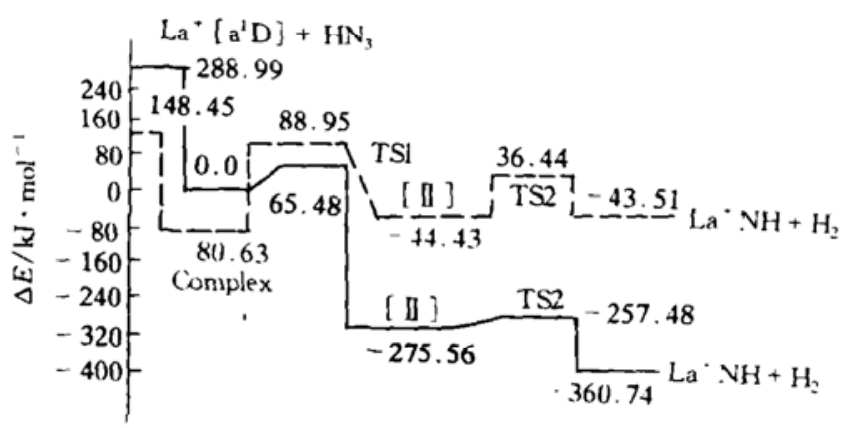

图 $1 \mathrm{La}^{+}+\mathrm{HN}_{3} \rightarrow \mathrm{La}^{+} \mathrm{NH}+\mathrm{H}_{2}$ 反应势能剖面示意图 实线为单态, 遮线为三重态, 将单态碰撞垎合物枧为零点, 相应总能 量为 -57.51482698 a.u. 


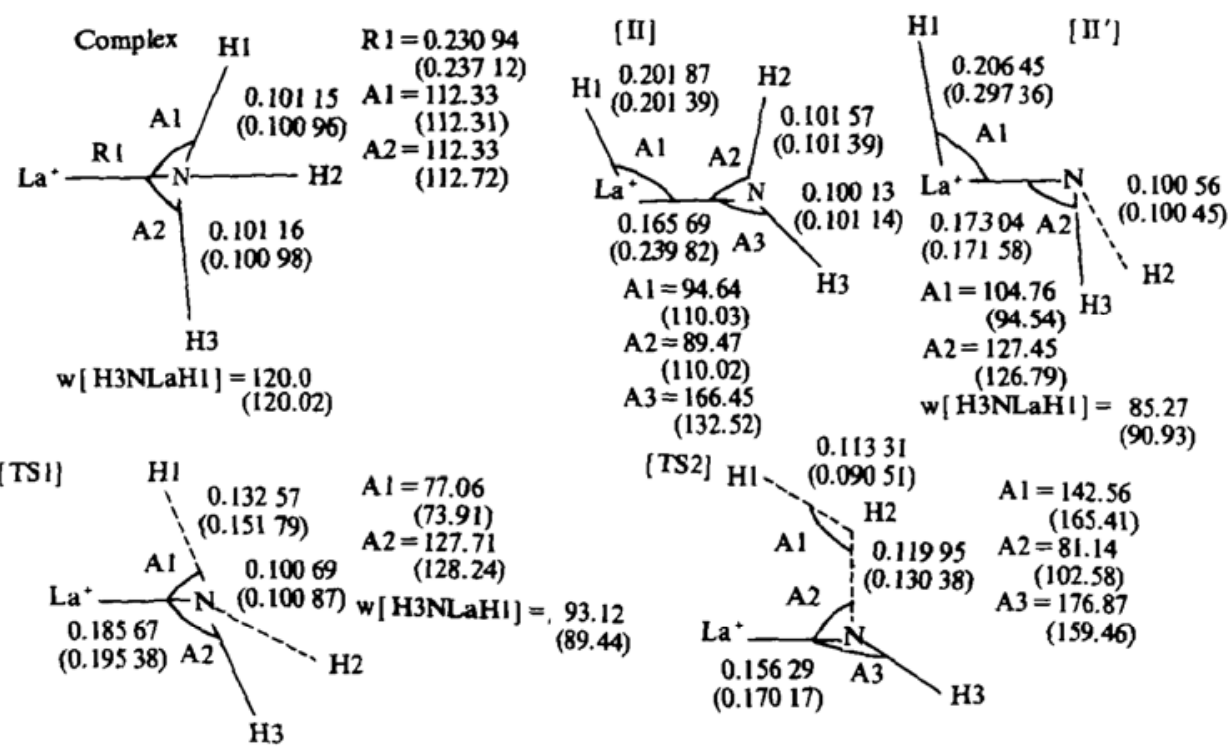

图 2 全优化所得稳定点及过渡态几何构型

键长用 $\mathrm{nm}$, 键角用“ $\left(^{\circ}\right)^{\circ}$ ”表示, 小括号内为相应三重态的构型参数

$\mathrm{N}$ 原子迁移到 $\mathrm{La}^{+}$形成中间体 $\mathrm{HLa}^{+}-\mathrm{NH}_{2}$ (II) 及 $\left.\mathrm{HLa}^{+} \mathrm{NH}_{2}(\mathrm{II})^{\prime}\right)$, 之后, 中间体 $\mathrm{H}-\mathrm{La}^{+}$ $\mathrm{NH}_{2}$ (II) 的 1,2- $\mathrm{H}_{2}$ 经过 4 中心过渡态 TS2 脱去, 得到产物 $\mathrm{La}^{+}-\mathrm{NH}+\mathrm{H}_{2}$.

碰撞络合物因 Jahn-Teller 效应 ${ }^{[9]}$ 具有 Cs 对称性, 而不是 $\mathrm{C}_{3 \mathrm{v}}$ 对称性, 与反应物的相对能 量为 $-288.99 \mathrm{~kJ} / \mathrm{mol}, \mathrm{La}^{+}-\mathrm{N}$ 键是由 $\mathrm{N}$ 原子的孤对电子与 $\mathrm{La}^{+}$的 $5 \mathrm{~d}$ 空轨道形成的配键, 键 长 $0.23034 \mathrm{~nm}$.

TS1 对应 $\mathrm{H} 1$ 原子从 $\mathrm{N}$ 迁移到 $\mathrm{La}^{+}$, 能垒为 $65.48 \mathrm{~kJ} / \mathrm{mol}$. 中间体 $\mathrm{H}-\mathrm{La}^{+}-\mathrm{HN}_{2}$ 有两个异 构体, 平面构型( II ) 及非平面构型 (II'), ( II ) 比 ( II ') 稳定 $43.81 \mathrm{~kJ} / \mathrm{mol}$. 对 $\mathrm{Sc}^{+}-\mathrm{NH}_{3}$ 体系, 平面中间体比非平面中间体稳定 $43.01 \mathrm{~kJ} / \mathrm{mol}^{[6]}$, 这主要由于在平面构型的 $\mathrm{H}^{-} \mathrm{La}^{+}-\mathrm{NH}_{2}$ ( II ) 中, 氮原子的孤电子对与 $\mathrm{La}^{+}$的 $5 \mathrm{~d}$ 空轨道间的 $\pi$ 重叠相互作用较非平面构型的强, 从而使其

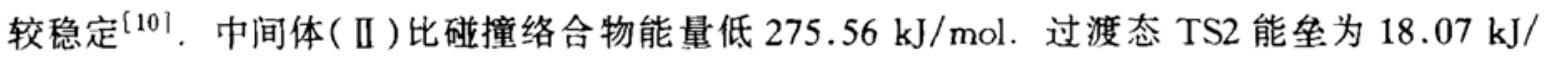
$\mathrm{mol}$. 由上可知, TS1 为速度控制步骤. 产物 $\mathrm{La}^{+} \mathrm{NH}+\mathrm{H}_{2}$ 比中间体 (II) 能量低 $85.19 \mathrm{~kJ} /$ $\mathrm{mol}$, 该途径有负的活化势垒 $-223.51 \mathrm{~kJ} / \mathrm{mol}$, 反应的焓变为 $-649.73 \mathrm{~kJ} / \mathrm{mol}$.

\section{2 单态及三态势能剖面比较}

在同一水平下优化所得三重态对应稳定点及过渡态结构见图 2. $\mathrm{La}^{+}$的基态为三重态 $\left(\mathrm{a}^{3} \mathrm{~F}\right)$, 其碰撞络合物比激发单态 $\left(\mathrm{a}^{1} \mathrm{D}\right)$ 的碰撞络合物稳定 $80.63 \mathrm{~kJ} / \mathrm{mol}$ (见图 1). 比较单态及 三重态的中间体 (II) 可看到: La-N 键长由单态的 0.16569 变为三重态的 $0.23982 \mathrm{~nm}$, 而中 间体 ( II') 中的 $\mathrm{H}-\mathrm{La}$ 键长由单态的 $0.20645 \mathrm{~nm}$ 变为三重态的 $0.29736 \mathrm{~nm}$. 在三重态 (II) 中, $\mathrm{La}^{+}-\mathrm{N}$ 之间的 Mulliken 原子重叠集居数仅为 0.10674 (单态 (II) 中 $\mathrm{La}^{+}-\mathrm{N}$ 之间 Mulliken 重叠集居数为 0.6046 ), 说明 $\mathrm{La}-\mathrm{N}$ 键由 $\mathrm{NH}_{3}$ 中氮原子的孤电子对与 $\mathrm{La}^{+}$的 $5 \mathrm{~d}$ 空轨道形成, $\mathrm{La}^{+}$的另一价电子对 $\mathrm{La}^{+}-\mathrm{N}$ 键设有贡献; 而在三重态 (II') 中, $\mathrm{H}-\mathrm{La}^{+}$之间的 Mulliken 重叠布 居数仅为 0.02928 , 说明 $\mathrm{H}-\mathrm{La}^{+}$之间未成键. 所以, 在三重态中间体 II 及 II '中, $\mathrm{La}^{+}$仅有一个 价电子是成键的, 另一个因高自旋而为非键电子. 由图1易看出, 三重态时速控制步骤仍为 
【研究简报】

\title{
气凝胶超微粉 $\mathrm{Fe} / \mathrm{ZrO}_{2}$ 的光声光谱研究
}

\author{
苏庆德 (1) 刘方新 ${ }^{(2)}$ 杨跃涛 赵天鹏 (3) 巨新 (4) 相宏伟 ${ }^{(4)}$
}

(1)中国科学技术大学应用化学系; (2)结构分析研究开放实验室; (3)物理系, 合肥 230026;

(4)中国科学院高能物理研究所, 北京 100039)

\section{关钺词 光声光㬐 气凝胶超微粉 $\mathrm{Fe} / \mathrm{ZrO}_{2} \quad$ 量子尺寸效应}

当固体材料的晶粒直径达到激子的 Pole 直径时, 其电子性质开始发生变化. 这种量子尺 寸效应使得材料显现出许多奇特的物理和化学性质, 引起了许多科学工作者的浓厚兴

$\mathrm{TS} 1$, 能垒为 $169.58 \mathrm{~kJ} / \mathrm{mol}$, 较之单态的能垒高 $104.09 \mathrm{~kJ} / \mathrm{mol}$, 反应焓变为 $-191.96 \mathrm{~kJ} / \mathrm{mol}$. 所以, 从热力学及动力学的角度而言, 该反应的单态途径为能量上最有利的途径, 当 $\mathrm{La}^{+}$为激 发单态时, 与中间体 $\mathrm{H}-\mathrm{La}^{+} \mathrm{NH}_{2}$ 及产物 $\mathrm{La}^{+} \mathrm{NH}$ 保持自旋一致, 反应活性高于基态 $\mathrm{La}^{+}$, 证实 了 Clemmer 等的“自旋守恒”概态的正确性.

一般而言, 激发态参与反应可能有两种作用, 一是增加反应的可资用能; 二是导致反应进 入不同的势能面. 本文所研究的 $\mathrm{La}^{+}$与 $\mathrm{NH}_{3}$ 反应适于后者, 即脱 $\mathrm{H}_{2}$ 反应必须经过一个插入 中间体 $\mathrm{H}-\mathrm{La}^{+}-\mathrm{NH}_{2}$ 后通过一个 4 中心过渡态由 1-2 脱 $\mathrm{H}_{2}$ 的方式脱去 $\mathrm{H}_{2}$. 而插入中间体 $\mathrm{H}-$ $\mathrm{La}^{+}-\mathrm{NH}_{2}$ 的基态是单态, 因此, 激发单态的 $\mathrm{La}^{+}$比基态三重态有更强的反应性, 整个过程保持 自旋守恒是个重要的概念.

致谢 本工作为国家自然科学基金(批准号:29273126)资助项目.

\section{参考文献}

1 Armentrout P B. In: Davies J A, Watson P L, Greenberg A, et al eds. Selective Hydrocarbon Activation: Principles; Progress. New York: VCH Pubishers, 1990. 467 533

2 Eller K, Schwarz H. Organometallic chemistry in the gas phase. Chem Rew, 1991, 91(6): 1 121 1 177

3 Ritter D, Weisshaar J C. Reactivity of neutral Fe, $\mathrm{Co}, \mathrm{Ni}$ and $\mathrm{Cu}$ atoms with Alkanes and Alkenes in the gas phase. J Am Chem Soc, 1990, 112: $6425 \sim 6426$

4 Clemmer D E, Sunderlin L S, Armentrout P B. Ammonia activation by $\mathrm{Sc}^{+}$and $\mathrm{Ti}^{+}$: Electrunic and translational energy dependence. J Phys Chem, 1990, 94(7): $3008 \sim 3015$

5 Clemmer D E, Sunderlin L S, Armentrout P B. Ammonia activation by $\mathrm{V}^{+}$: Electronic and translational energy dependence. J Phys Chem, 1990, 94(1): 208 217

6 Song Y. ab initio study on the reaction $\mathrm{Sc}^{+}+\mathrm{NH}_{3} \rightarrow \mathrm{Sc}^{+} \mathrm{NH}+\mathrm{H}_{2}$. Mol Struct (Theochem), 1995, 357(82): $147 \sim 152$

7 Ross R B, Powers J M, Ermler W C, et al. ab initio relativistic effective potentials with spin-orbit operators. IV . Cs through Rn. J Chem Phys, 1990, 93(9): $6654 \sim 6670$

8 Schmidt M W, Baldridge K K, Boatz J A, et al. GAMESS (General atomic and molecular electronic structure system). QCPE Bulletin, 1990, 10: 52 -54

9 Song Y. Ground and excited state reaction of $\mathrm{N}^{+}$with ammonia. J Mol Struct (Theochem), 1993, 283: 261 265

10 Raban M. Aviran K, Kost D. The three electron $\pi$-bond in hydrazyl radical. An ab initio SCF-MO investigation of torsional and inversional stereomutation. Tetrahedron Lett, 1985, 26(30): $3591 \sim 3594$

(1996-02-26 收稿, 1996-09-03 收修改稿) 